\title{
A cross-sectional and short-term longitudinal characterisation of NIDDM in Psammomys obesus
}

\author{
M. Barnett ${ }^{1}$, G. R. Collier ${ }^{1}$, F. McL. Collier ${ }^{1}$, P.Zimmet ${ }^{2}$, K. O'Dea ${ }^{1}$ \\ ${ }^{1}$ School of Nutrition and Public Health, Deakin University, Victoria, Australia \\ ${ }^{2}$ International Diabetes Institute, Caulfield General Medical Centre, Caulfield South, Victoria, Australia
}

\begin{abstract}
Summary The present study was undertaken to examine the cross-sectional and short-term longitudinal changes in glucose and insulin concentrations as well as measure the enzymatic activity of PEPCK and glycogen synthase in our Psammomys obesus colony. In the cross-sectional study, blood samples were taken from one group of animals at 19 weeks of age $(n=37)$ in the fed state and following a 4-h fast. In a separate group of 19-week-old animals $(n=69)$, samples were taken $1 \mathrm{~h}$ following an OGTT ( $1 \mathrm{~g} / \mathrm{kg}$ body weight) in Psammomys subjected to a 16 -h fast. In the longitudinal study, blood samples were taken from one group of animals in the fed state at 7,11,15 and 19 weeks of age. All of the cross-sectional data have described the classic inverted U-shaped curve (Starling's curve of the pancreas) in the relationship between glucose and insulin levels. This trend was also reflected by Psammomys subjected to the OGTT; a mild impairment in glucose tolerance was associated with an increase in the insulin response and a further impairment in glucose tolerance was associated with a reduction in the insulin response. Similar results were obtained following a 4-h fast. The short-term longitudinal glucose and insulin data revealed that of the 37 animals examined over the 12 -week period, 16 progressed along the inverted $U$ -
\end{abstract}

shaped curve described by the cross-sectional data. Of the other animals, 8 remained unchanged, 7 were unclassifiable and 6 hyperglycaemic Psammomys developed normoglycaemia at the expense of elevated insulin levels. Psammomys were divided into four separate groups based on their glucose and insulin levels in the fed state at age 19 weeks: group A were normoglycaemic $(4.5 \pm 0.2 \mathrm{mmol} / \mathrm{l})$ and normoinsulinaemic $(81 \pm 15 \mathrm{mU} / \mathrm{l})$; group B were normoglycaemic $(5.3 \pm$ $0.3 \mathrm{mmol} / \mathrm{l})$ and hyperinsulinaemic $(267 \pm 19 \mathrm{mU} / \mathrm{l})$; group $C$ were hyperglycaemic $(16.9 \pm 1.4 \mathrm{mmol} / \mathrm{l})$ and hyperinsulinaemic $(356 \pm 29 \mathrm{mU} / 1)$; group D were hyperglycaemic $(20.4 \pm 1.4 \mathrm{mmol} / \mathrm{l})$ and normoinsulinaemic $(92 \pm 56 \mathrm{mU} / \mathrm{l})$. Analysis of liver and soleus muscle tissue revealed that active glycogen synthase activity was reduced in the hyperglycaemic Psammomys (C and D). Total glycogen synthase activity (in liver and muscle) and PEPCK activity (in liver) were not different between groups A-D. These findings add to those already published which suggest Psammomys is an ideal animal model for the study of NIDDM. [Diabetologia (1994) 37: 671-676]

Key words Psammomys obesus, glucose, insulin, crosssectional, longitudinal, PEPCK, glycogen snythase.
Received: 19 November 1993

and in revised form: 22 February 1994

Corresponding author: Dr. G. R. Collier, School of Nutrition and Public Health, Deakin University, Victoria, 3217, Australia

Abbreviations: NIDDM, Non-insulin-dependent diabetes mellitus; OGTT, oral glucose tolerance test; PEPCK, phosphoenolpyruvate carboxykinase.
The development of hyperglycaemia in Psammomys obesus following ad libitum feeding of laboratory chow was first demonstrated in 1964 [1]. In conjunction with hyperglycaemia, other physiological defects have been reported including hyperinsulinaemia, reduced glucose tolerance, obesity, cataracts and betacell degranulation and vacuolisation of the pancreatic islet tissue $[1,2]$. The complications presented in these pathology studies in conjunction with the wide range of glycaemic response to a chow diet made Psammo- 


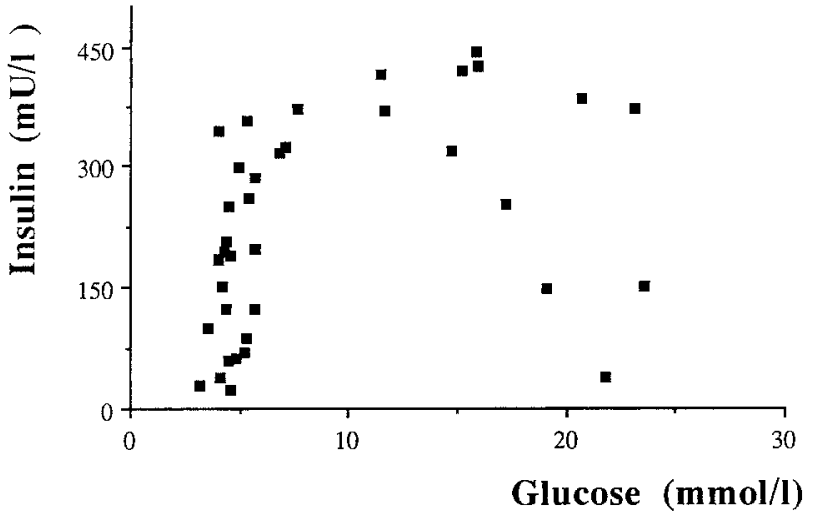

Fig. 1. The inverted $U$-shaped curve relationship describing the plasma glucose and insulin concentrations of 3719 -week-old Psammomys in the fed state
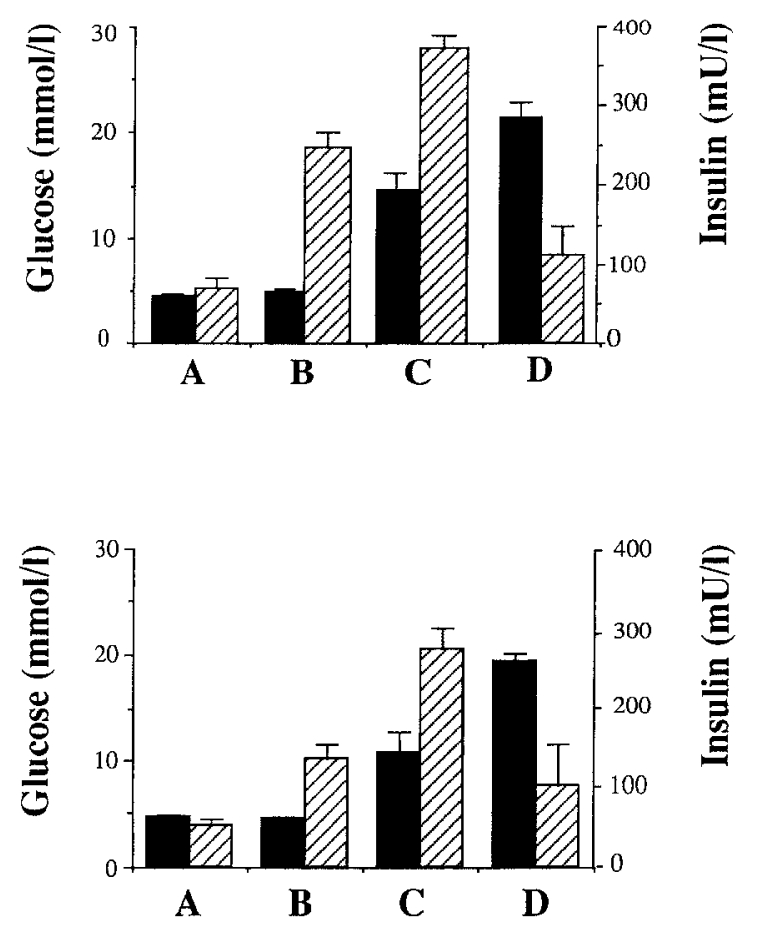

Fig. 2. Plasma glucose ( $\square$ ) and insulin ( $\square$ ) concentrations of four separate groups of 19-week-old Psammomys in the fed state (upper panel) or following a 4-h fast (lower panel): Group A was normoglycaemic and normoinsulinaemic $(n=10)$, group $\mathrm{B}$ was normoglycaemic and hyperinsulinaemic $(n=13)$, group $C$ was hyperglycaemic and hyperinsulinaemic $(n=11)$ and group D was hyperglycaemic and normoinsulinaemic $(n=3)$. Results are expressed as means \pm SEM

mys an ideal animal model for the study of NIDDM. This range of glycaemic response to a chow diet enabled a Psammomys colony to be characterised by the glucose and insulin levels in the fed state [3]. Four groups of animals were identified; group A (normoglycaemic, normoinsulinaemic), group B (normoglycaemic, hyperinsulinaemic), group C (hyperglycaemic, hyperinsulinaemic) and group D (hyperglycaemic, normoinsulinaemic). Longitudinal data have also been presented although glucose was the only metabolite measured [4].

Unfortunately, in these cross-sectional and longitudinal studies, Psammomys were not matched for age. In addition, blood samples were only taken in the fed state which suggested the possibility that the variation in glucose and insulin response may have been partly due to variation in the time period between the last meal and the collection of the blood sample. Surprisingly, in light of the aforementioned studies, longitudinal glucose and insulin data describing the progressive changes during the development of diabetes are yet to be presented on this animal model of NIDDM. Despite the difficulties associated with breeding Psammomys [5], we have successfully established a breeding colony at Deakin University.

In the present study we examined glucose and insulin levels cross-sectionally in 37 19-week-old Psammomys in the fed state and following a 4-h fast, and in 69 Psammomys, $1 \mathrm{~h}$ after an OGTT, which was preceded by a 16 -h fast $(n=69)$. We also measured glucose and insulin levels longitudinally over a 12-week period. In addition, PEPCK and glycogen synthase activities were measured in liver and muscle tissue of 19 week-old animals in the fed state.

\section{Materials and methods}

Breeding. Psammomys from our colony were bred at Deakin University using the San Poiley method [6] to maximise the size of the genetic pool. Breeders were given free access to a diet consisting of standard laboratory chow (Baristoc, Pakenham, Victoria, Australia) and spinach. The energy components of the chow pellets comprised $12 \%$ energy from fat, $63 \%$ energy from carbohydrate and $25 \%$ energy from protein. Animals were weaned at 3 weeks of age, fed chow ad libitum and were housed in groups of three to five in a temperature controlled room $\left(20^{\circ} \mathrm{C}\right)$ with a $12-12 \mathrm{~h}$ light-dark cycle. The colony has been maintained in accordance with the guidelines of the National Health and Medical Research Council [7].

Cross-sectional and short-term longitudinal studies. In all the cross-sectional studies, male Psammomys were examined at 19 weeks, which is an age that is associated with a wide variation in glycaemic response ranging from normal to impaired glucose tolerance to NIDDM. In one group of Psammomys $(n=37)$, glucose and insulin levels were measured in the fed state and following a 4-h fast. In a second group $(n=69)$, the glucose and insulin response was measured $1 \mathrm{~h}$ following an OGTT ( $1 \mathrm{~g} / \mathrm{kg}$ body weight) which was preceded by a 16 -h fast. In the longitudinal study, $(n=37)$, blood samples were taken 7 , 11,15 and 19 weeks of age in the fed state to measure glucose and insulin levels.

Analytical methods. All blood samples were taken in the morning by tail tipping, and were collected in heparinized tubes and placed on ice. Samples were centrifuged to separate the plasma which was stored at $-20^{\circ} \mathrm{C}$ for subsequent analysis. Plasma glucose concentrations were determined on an automatic analyser (Hitachi, 705-0013, Tokyo, Japan) using a colorimetric method (Boehringer, Mannheim, Mannheim, Germany). Insulin concentrations were determined against a standard of human in- 
Table 1. The activities of PEPCK and glycogen synthase in liver and muscle tissue of Psammomys in the fed state that were 19 weeks of age

\begin{tabular}{llcccc}
\hline $\begin{array}{l}\text { Enzyme Activity } \\
\text { (nmol· } \mathrm{min}^{-1} \mathrm{mg}^{-1} \text { of tissue) }\end{array}$ & Tissue & Group A & Group B & Group C & Group D \\
\hline PEPCK & Liver & $0.46 \pm 0.05$ & $0.42 \pm 0.04$ & $0.49 \pm 0.02$ & $0.59 \pm 0.17$ \\
Act. Gly. Syn. & Liver & $0.064 \pm 0.01$ & $0.049 \pm 0.005$ & $0.028 \pm 0.003^{\mathrm{a}}$ & $0.032 \pm 0.001^{\mathrm{a}}$ \\
Tot. Gly. Syn. & Liver & $0.33 \pm 0.08$ & $0.30 \pm 0.04$ & $0.33 \pm 0.06$ & $0.25 \pm 0.01$ \\
Act. Gly. Syn. & Soleus & $0.80 \pm 0.08$ & $0.68 \pm 0.06$ & $0.59 \pm 0.08^{\mathrm{a}}$ & $0.44 \pm 0.04^{\mathrm{a}}$ \\
Tot. Gly. Syn. & Soleus & $2.95 \pm 0.24$ & $3.11 \pm 0.22$ & $2.73 \pm 0.44$ & $2.89 \pm 0.27$ \\
Act. Gly. Syn. & Red Gast. & $0.72 \pm 0.06$ & $0.62 \pm 0.05$ & $0.58 \pm 0.09^{\mathrm{a}}$ & $0.56 \pm 0.13^{\mathrm{a}}$ \\
Tot. Gly. Syn. & Red Gast. & $3.06 \pm 0.09$ & $2.96 \pm 0.33$ & $2.62 \pm 0.48$ & $1.36 \pm 0.47$ \\
Act. Gly. Syn. & White Gast. & $0.41 \pm 0.07$ & $0.49 \pm 0.06$ & $0.51 \pm 0.06$ & $0.63 \pm 0.09$ \\
Tot. Gly. Syn. & White Gast. & $2.76 \pm 0.09$ & $2.87 \pm 0.34$ & $2.51 \pm 0.28$ & $2.88 \pm 0.27$ \\
\hline
\end{tabular}

All values are expressed as means \pm SEM for group $\mathrm{A}(n=5)$, group $\mathrm{B}(n=7)$, group $\mathrm{C}(n=5)$ and group $\mathrm{D}(n=4)$. The superscript 'a' denotes a difference compared to group $\mathrm{A}(p<0.05)$

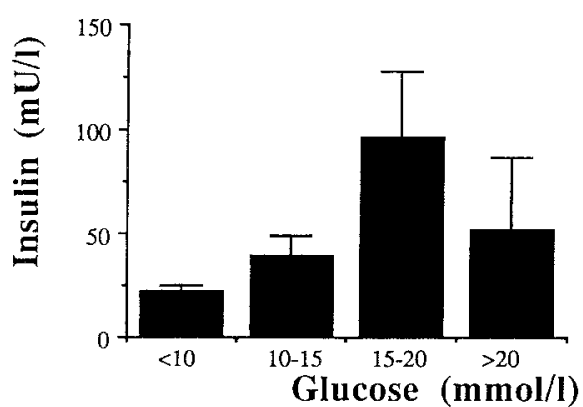

Fig.3. Plasma glucose and insulin responses $1 \mathrm{~h}$ after oral glucose $(1 \mathrm{~g} / \mathrm{kg}$ body weight) which was preceded by a $16-\mathrm{h}$ fast in four groups of 19-week-old Psammomys: Group A was normoglycaemic and normoinsulinaemic $(n=30)$, group B was normoglycaemic and hyperinsulinaemic $(n=27)$, group $\mathrm{C}$ was hyperglycaemic and hyperinsulinaemic $(n=9)$ and group $D$ was hyperglycaemic and normoinsulinaemic $(n=3)$. Results are expressed as means \pm SEM

sulin using a double antibody radioimmunoassay (Pharmacia Diagnostics, Uppsala, Sweden).

Animals were killed by cervical dislocation. Liver tissue was immediately freeze-clamped and then frozen in liquid nitrogen. The skeletal muscle tissue removed from each animal comprised the slow twitch red fibers with a high oxidative capacity (soleus and red gastrocnemius) and the fast twitch white fibers with a low oxidative capacity (white gastrocnemius). Tissues were rapidly frozen in liquid nitrogen and stored at $-70^{\circ} \mathrm{C}$ for subsequent analysis.

PEPCK activity was measured in liver tissue and estimated using the oxaloacetate exchange assay [8]. Active and total glycogen synthase activity was measured in liver, soleus muscle and white and red gastrocnemius muscle $[9,10]$.

\section{Statistical analysis}

All experimental data have been expressed as means \pm SEM. A one-way analysis of variance was used in conjunction with a Tukey's multiple range test to determine any differences in glucose and insulin levels, and enzymatic activity between the Psammomys groups. Probability values less than 0.05 were considered significant.

\section{Results}

Cross-sectional characterisation. An inverted Ushaped curve (Fig.1) described the relationship between plasma glucose and insulin levels. A small increase in glucose levels was associated with a sharp increase in insulin levels. A further increase in the glucose concentration ( $>15 \mathrm{mmol} / \mathrm{l}$ ) was associated with a general decline in insulin levels despite marked hyperglycaemia.

Psammomys were categorised into four distinct groups (A-D) according to their plasma glucose and insulin levels in the fed state and following a 4-h fast (Fig.2). Animals were selected for each category on the basis of hyperglycaemia being defined as glucose levels of $8 \mathrm{mmol} / \mathrm{l}$ or more and hyperinsulinaemia being defined as insulin levels of $150 \mathrm{mU} / 1$ or more. The four groups were as follows: group A $(n=10)$ were normoglycaemic ( $\leq 8 \mathrm{mmol} / \mathrm{l})$ and normoinsulinaemic $(\leq 150 \mathrm{mU} / \mathrm{l})$, group B $(n=13)$ were normoglycaemic $(\leq 8 \mathrm{mmol} / \mathrm{l})$ and hyperinsulinaemic $(\geq 150 \mathrm{mU} / \mathrm{l})$, group C $(n=11)$ were hyperglycaemic $(\geq 8 \mathrm{mmol} / \mathrm{l})$ and hyperinsulinaemic $(\geq 150 \mathrm{mU} / \mathrm{l})$ and group $\mathrm{D}$ $(n=3)$ were hyperglycaemic $(\geq 8 \mathrm{mmol} / \mathrm{l})$ and normoinsulinaemic ( $\leq 150 \mathrm{mU} / \mathrm{l})$. Animals that were classified into their respective groups in the fed state remained in the same groups when glucose and insulin levels were measured following a 4-h fast.

Enzymatic analysis of liver and muscle tissue (Table 1) showed that active glycogen synthase activity was reduced in the liver and soleus muscle of the hyperglycaemic Psammomys (groups C and D) when compared to group $\mathrm{A}(p<0.05)$. There were no differences between groups in the activities of total glycogen synthase and PEPCK in either liver, soleus muscle or gastrocnemius muscle (white and red).

In a separate group of Psammomys $(n=69)$ subjected to a 16-h fast, animals were categorised into four groups according to the plasma glucose concentrations $1 \mathrm{~h}$ following the OGTT (Fig. 3). Animals with a relatively low glucose response (glucose $\leq 10 \mathrm{mmol} / 1$, $n=30$ ) also demonstrated a relatively low insulin re- 


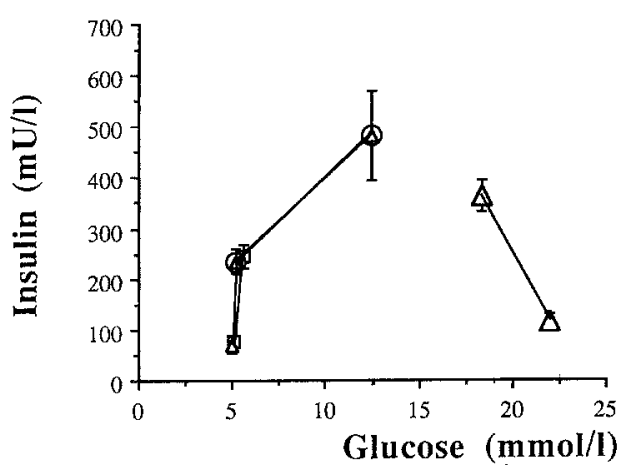

Fig.4. Short-term longitudinal trends in plasma glucose and insulin levels (in the fed state) in Psammomys ranging from 7 to 19 weeks of age. The longitudinal changes indicated four groups of Psammomys that followed separate parts of the inverted ' $U$ ' shaped curve within a 12 -week period: $\mathrm{A}$ to $\mathrm{B}(\square) n=11, \mathrm{~B}$ to $\mathrm{C}$ (O) $n=5$, C to $\mathrm{D}(\triangle) n=5$, and $\mathrm{A}$ to $\mathrm{B}$ to $\mathrm{C}(\Delta) n=5$. Results are expressed as means \pm SEM. Groups A, B, C, and D are defined as in Figure 2

sponse. A progressive increase in the insulin response was associated with an increase in the glucose response as demonstrated by the animals with mild or moderately impaired glucose tolerance ( $n=27$ and $n=9$, respectively). A further deterioration of glucose tolerance ( $n=3$ for glucose $\geq 20 \mathrm{mmol} / \mathrm{l}$ ) was associated with reduced insulin levels.

Short-term longitudinal study. From the 37 animals that were followed-up longitudinally, 16 demonstrated changes in plasma glucose and insulin levels that followed separate parts of the inverted U-shaped curve (Fig. 4) similar to that described in the cross-sectional data. The transition from group A to group B was associated with an increase in insulin levels ( $76 \pm 9$ vs $243 \pm 23 \mathrm{mU} / \mathrm{l}, n=11, p<0.05$ ) with a minor significant increase in plasma glucose levels $(5.1 \pm 0.2$ vs $5.6 \pm$ $0.2 \mathrm{mmol} / \mathrm{l})$. The transition from group B to group C was associated with significant increases in both plasma glucose levels $(5.2 \pm 0.2$ vs $12.4 \pm 1.5 \mathrm{mmol} / \mathrm{l}, n=5$, $p<0.05)$ and plasma insulin levels $(235 \pm 26$ vs $483 \pm 88 \mathrm{mU} / 1, p<0.05)$. In the transition from group $\mathrm{C}$ to group D, plasma insulin levels dropped dramatically despite continued hyperglycaemia (362 \pm 31 vs $113 \pm 16 \mathrm{mU} / 1, n=5, p<0.05$ ). A total of five animals progressed from group $\mathrm{A}$ to group $\mathrm{B}$ to group $\mathrm{C}$, and the changes in plasma glucose and insulin levels were similar to the changes just described. During the 12week period of the longitudinal study, only one animal progressed from group A through to group D. Of the Psammomys that did not follow the longitudinal trends just described, eight did not change groups, seven were unclassifiable as they demonstrated random changes that were not consistent with any particular trend and six that were initially hyperglycaemic improved to become normoglycaemic at the expense of elevated insulin levels.

\section{Discussion}

The inverted U-shaped curve has described a relationship between fed glucose and insulin levels which has not been presented before in this model of NIDDM. It is similar to data presented previously in human studies that have examined the relationship between glucose and insulin concentrations (fasting or in response to a glucose load) in a cross-section of subjects including normal weight control subjects, individuals with impaired glucose tolerance and in NIDDM subjects with varying degrees of fasting hyperglycaemia [11-13]. As the fasting glucose levels increased within the crosssectional data, there was a progressive increase in insulin levels. A further increase in glucose levels was associated with a rapid decline in insulin levels. De Fronzo [14] has coined this relationship 'Starling's curve of the pancreas'.

Psammomys could be divided into four separate groups based on the cross-section of glucose and insulin levels in the fed state. These results are consistent with a previous classification of Psammomys that measured the fed glucose and insulin levels in the Psammomys which ranged from 1 to 20 months of age [3]. As it was not clear from this study whether the variation in glucose and insulin levels between the groups was an effect due to the age range of the animals and/or the variation in the time period between the last meal and collection of the blood sample, we controlled for both of these variables in the current study. Not only were the animals matched for age, but the fed cross-sectional data were duplicated when all Psammomys were subjected to a 4-h fast.

A previous study involving the rhesus monkey has reported cross-sectional data based on glucose and insulin concentrations following a 16-h fast [15]. A total of nine phases were described. Phase 1 had normal glucose and insulin levels, while in the transition from phase 2 to phase 7 , insulin levels gradually increased despite the glucose levels remaining normal. Glucose levels increased significantly from phase 8 to phase 9 although this was associated with a corresponding reduction in insulin levels. These cross-sectional results which conform to those presented here are similar to the human cross-sectional data described above and suggest that the Psammomys is an appropriate animal model with which to examine the aetiology of NIDDM.

When the Psammomys were subjected to a $1-\mathrm{h}$ OGTT it was still possible to categorise the animals into discrete groups based on differing glucose tolerance. However, it is interesting to note that the insulin response to the glucose load was significantly lower than that to chow. This may be related to prolonged fasting period $(16 \mathrm{~h})$ that preceded the glucose load, or it may indicate that secretagogues other than glucose are important in the development of hyperinsulinaemia in the Psammomys when it is deprived of its 
natural diet of salt bush and placed on a diet of laboratory chow which has greater concentrations of fat and protein [16]. A previous study in human subjects has demonstrated the potentiating effect of fat on glucosestimulated insulin secretion [17]. In the Psammomys which are genetically predisposed to NIDDM, it is possible that an elevated insulin response to the chow diet leads to a further exacerbation of insulin resistance which together contribute to the development of NIDDM. The adverse effects of hyperinsulinaemia have been well documented [18]. The administration of insulin to healthy subjects for several hours to a few days resulted in insulin resistance as measured by the euglycaemic-insulin clamp, in conjunction with a reduction in insulin binding [19]. Collectively, these findings may explain at least in part the high prevalence of NIDDM in genetically predisposed human populations that have changed from their traditional diets to 'Western' styled diets which are often higher in fat. Not surprisingly, hyperinsulinaemia has been found to be a predictor of NIDDM even in the adolescents of such populations as the Micronesian Naurans and Polynesian Tuvaluans [20]. However, a further progression towards the development of NIDDM in these genetically predisposed populations is accompanied by pancreatic decompensation resulting in reduced insulin levels $[12$, 21]. This pattern of distribution of glucose and insulin levels has been demonstrated by the Psammomys in the present study. The development of hypoinsulinaemia in the presence of worsening hyperglycaemia is consistent with the phenomenon of 'glucotoxicity' and may be responsible for the deterioration of pancreatic insulin secretion $[14,22]$.

The enzymatic analysis in this study has suggested that a defect in active glycogen synthase and not PEPCK is in part responsible for the hyperglycaemia associated with this model. Previous investigations have estimated glycogen synthesis in the Psammomys by measuring the rate at which labelled glucose is incorporated into glycogen, and demonstrasted impaired glycogen synthesis in both the basal and insulin-stimulated states for rats classified in groups B, C and D [3, $23]$. These results alone suggest that reduced glycogen synthesis may be an earlier predictor for insulin resistance than elevated gluconeogenesis. This is consistent with the findings presented in two human studies that have exposed glycogen synthase activity defects, and more importantly, defects in the glycogen synthase gene in subjects with NIDDM $[24,25]$. Furthermore, when compared to group A Psammomys, 2-deoxy glucose uptake in the muscle was reduced in group B and further reduced in group $\mathrm{C}$ animals, while the activity of PEPCK, was reduced in group B and normalised in group C Psammomys [3]. Similar trends were demonstrated for PEPCK in the present study although the results failed to reach statistical significance. It should be noted that the Psammomys in that particular study were not matched for age and this may possibly account for any differences in PEPCK measurements between the two studies.

Although the cross-sectional data in this study have demonstrated a specific relationship between glucose and insulin levels, it has also suggested a possible pattern in the development of NIDDM. Only the longitudinal data can serve to confirm whether the glucose and insulin changes in the development of NIDDM occur in the pattern suggested by the cross-sectional data.

In the short-term longitudinal study where glucose and insulin levels were measured in the fed state, the data has indicated that of the 37 rats studied, 16 progressed along separate portions ( $\mathrm{A}$ to $\mathrm{B}, \mathrm{B}$ to $\mathrm{C}, \mathrm{C}$ to $\mathrm{D}$, $A$ to $B$ to $C$ and $A$ to $B$ to $C$ to $D$ ) of an inverted $U$ shaped curve. These results are encouraging in light of the relatively brief period ( 12 weeks) of the longitudinal study. These trends are consistent with the data from a 6-year longitudinal study on the rhesus monkey [26]. Seven monkeys developed diabetes through the sequence of glucose and insulin changes suggested by their cross-sectional data which was similar to the data presented here; however, 14 of the monkeys did not. Unfortunately, the longitudinal trends that these monkeys followed were not described by the authors and so no comparison can be made between the two studies. Perhaps the failure of some of the Psammomys and rhesus monkeys to completely follow the longitudinal trends (i.e. normal to diabetic monkeys) suggested by their respective cross-sectional data may be due to the relatively short duration of these longitudinal studies. Our study in particular, only followed the Psammomys for 12 weeks. The time period required for this longitudinal study may need to be extended to reveal trends consistent with the cross-sectional data in the majority of Psammomys. Furthermore, not all Psammomys are genetically predisposed to the development of NIDDM when subjected to a chow diet [3]. In addition, it is possible that the improvement demonstrated in six hyperglycaemic Psammomys that became normoglycaemic may have been associated with a reduction in energy intake.

The difficulties associated with conducting studies on the development of NIDDM within a practical time frame have been made apparent in extensive longitudinal human study (14 years) that followed the progression of glucose and insulin levels in 132 subjects of varying glucose tolerance [27]. Of these, only 11 individuals demonstrated the full cycle of changes (i. e. normal to diabetic) in glucose and insulin levels consistent with the pattern of the inverted U-shaped curve described earlier. However, the validity of any conclusions on the development of NIDDM drawn from cross-sectional data was strengthened by three other groups that were followed longitudinally (normal to impaired glucose tolerance, impaired glucose tolerance to NIDDM, and NIDDM). Each group reflected glucose and insulin changes consistent with separate parts of the inverted 
U-shaped curve. These intermediate longitudinal trends are similar to those described in the present study and further support the notion of the Psammomys being an appropriate animal model for the longitudinal study on the aetiology of NIDDM.

Acknowledgements. This work was in part supported by an internal Deakin University research grant and the Leon Mow Research Trust.

\section{References}

1. Schmidt-Nielsen K, Haines KB (1964) Diabetes mellitus in the sand rat induced by standard laboratory diets. Science 143: 689-690

2. Hackel DB, Schmidt-Nielsen K, Haines H, Mikat E (1965) Diabetes mellitus in the sand rat (Psammomys obesus): pathologic studies. Lab Invest 14: 200

3. Kalderon B, Gutman A, Levy E, Shafrir E, Adler JH (1986) Characterization of stages in development of obesitydiabetes syndrome in sand rat (Psammomys obesus). Diabetes 6:717-724

4. Adler JH, Lazarovici G, Marton M et al. (1988) Patterns of hyperglycemia, hyperinsulinemia and pancreatic insufficiency in sand rats (Psammomys obesus). In: Shafrir E, Renold AE (eds) Frontiers in diabetes research. Lessons from animal diabetes II. pp 384-388

5. Prange HD, Schmidt-Nielsen K, Hackel DB (1968) Care and breeding of the fat sand rat (Psammomys obesus Cretzschmar). Lab Anim Care 18: 170-181

6. Poiley SM (1960) A systematic method of breeder rotation for non-inbred laboratory animal colonies. Proceedings of the Animal Care Panel 10: 159-166

7. National Health and Medical Research Council (1990) Australian code of practice for the care and use of animals for scientific purposes. Australian Government Publishing Service

8. Chang HC, Lane MD (1966) The enzymatic carboxylation of phosphoenolpyruvate. J Biol Chem 211: 2413-2420

9. Golden S, Wals PA, Katz J (1977) An improved procedure for the assay of glycogen synthase and glycogen phosphorylase in rat liver homogenates. Anal Biochem 77: 436-445

10. Kruszynska YT, Home PD, Alberti KGMM (1986) In vivo regulation of liver and skeletal muscle glycogen synthase activity by glucose and insulin. Diabetes $35: 662-667$

11. Reaven G, Miller R (1968) Study of the relationship between glucose and insulin responses to an oral glucose load in man. Diabetes 70: 560-569

12. Zimmet P, Whitehouse S, Kiss J (1979) Ethnic variability in the plasma insulin response to oral glucose in Polynesian and Micronesian subjects. Diabetes 28: 624-628
13. DeFronzo RA, Ferrannini F, Simonson DC (1989) Fasting hyperglycemia in non-insulin-dependent diabetes mellitus: contributions of excessive hepatic glucose production and impaired tissue glucose uptake. Metabolism 38: 387-395

14. De Fronzo RA (1988) The triumvirate: B cell, muscle, liver A collusion responsible for NIDDM. Diabetes 37: 667-687

15. Bodkin NL, Metzger BL, Hansen BC (1989) Hepatic glucose production and insulin sensitivity preceding diabetes in monkeys. Am J Physiol 256: E676-E681

16. Shafrir E (1989) Diabetes in animals. In: H. Rifkin, D. Porte (eds) Diabetes mellitus. pp. 299-340

17. Collier GR, Greenberg GR, Wolever TMS, Jenkins DJA (1988) The acute effect of fat on insulin secretion. J Clin End Metab 66: 323-326

18. Reaven GM (1993) Role of insulin resistance in human diseaes (syndrome X): an expanded definition. Annu Rev Med 44: 121-131

19. Rizza RA, Mandarino LJ, Genest J, Baker BA, Gerich JE (1985) Production of insulin resistance in hyperinsulinemia in man. Diabetologia 28: 70-75

20. Zimmet PZ, Collins VR, Dowse GK, Knight LT (1992) Hyperinsulinaemia in youth is a predictor for type 2 (non-insulin-dependent) diabetes mellitus. Diabetologia 35: 534541

21. Arnoff SL, Bennett PH, Gordon P, Rushforth N, Miller M (1977) Unexplained hyperinsulinemia in normal and 'prediabetic' Pima Indians compared with normal Caucasians. An example of racial differences in insulin secretion. Diabetes 26: $827-840$

22. Leahy JL (1990) Natural history of B-cell dysfunction in NIDDM. Diabetes Care 13: 992-1010

23. Shulman GI, Rothman DL, Jue T, Stein P, De Fronzo RA, Shulman RG (1990) Quantitation of muscle glycogen synthesis in normal subjects, and subjects with non-insulin-dependent diabetes by ${ }^{13} \mathrm{C}$ nuclear magnetic resonance spectroscopy. New Eng J Med 322: 223-228

24. Groop LC, Kankuri M, Schalin-Jantti C et al. (1993) Association between polymorphism of the glycogen synthase gene and non-insulin-dependent diabetes mellitus. New Eng J Med 328: 10-14

25. Hansen BC, Bodkin NL (1986) Heterogeneity of insulin responses: phases leading to type 2 (non-insulin-dependent) diabetes mellitus in the rhesus monkey. Diabetologia 29: 713-719

26. Saad MF, Pettitt DJ, Mott DM, Knowler WC, Nelson RG, Bennett PH (1989) Sequential changes in serum insulin concentration during development of non-insulin-dependent diabetes. Lancet I: $356-1358$ 